\title{
MINERAÇÃO DO AMIANTO: UMA ABORDAGEM AOS RISCOS TECNOLÓGICOS AMBIENTAIS E SAÚDE DO TRABALHO
}

\section{ARTIGO ORIGINAL}

NUNES, Antônio José Ribeiro ${ }^{1}$

NUNES, Antônio José Ribeiro. Mineração do amianto: uma abordagem aos riscos tecnológicos ambientais e saúde do trabalho. Revista Científica Multidisciplinar Núcleo do Conhecimento. Ano 06, Ed. 06, Vol. 04, pp. 53-63. Junho de 2021. ISSN: 2448-0959, Link de acesso: https://www.nucleodoconhecimento.com.br/engenharia-ambiental/tecnologicosambientais, $\quad$ DOI: $\quad$ 10.32749/nucleodoconhecimento.com.br/engenhariaambiental/tecnologicos-ambientais

\section{RESUMO}

O Brasil é um dos grandes produtores mundiais de amianto ou asbesto, este se constitui em um mineral, cuja fibrilha natural tem sedosidade e propriedades físicas e químicas de grande valor para a indústria automobilística e de construção civil, devido à sua resistência mecânica e capacidade de suportar altas temperaturas. O amianto possui uma alta periculosidade para a saúde humana, com efeitos devastadores. A mineração no Estado de São Paulo e Rio de Janeiro foi proibida, em face dos problemas de trabalhadores afetados pela asbestose, mas diante de sua larga escala de uso na indústria nacional, a maioria das regiões brasileiras continua com as atividades de mineração. A finalidade desse artigo é demonstrar os riscos de natureza tecnológica e ambiental nas atividades produtivas contemporâneas da mineração de amianto. Pretende-se demonstrar os riscos iminentes das operações, os efeitos no organismo humano em trabalhadores com

\footnotetext{
${ }^{1}$ Pós Graduado - Auditoria, Gestão e Perícia Ambiental / Engenharia de Segurança do Trabalho; Graduado Engenharia de Minas.
}

RC: 87951

Disponível em: https://www.nucleodoconhecimento.com.br/engenharia- 
exposições prolongadas; demonstrar as doenças ocupacionais que decorrem da exposição contínua ao mineral. Os resultados demonstram que uso em larga escala em amplos setores de produção determina o seu uso aplicando-se medidas restritivas e limites de exposição do trabalhador. Mesmo assim, essa questão é ainda polêmica, considerando que São Paulo e Rio de Janeiro aboliram o uso do amianto diante dos efeitos negativos na saúde dos trabalhadores. A Confederação Nacional dos Trabalhadores da Indústria - CNTI tem buscado alternativas diante da importância do amianto na economia nacional cujas tendências são a extração controlada e com inspeção com métodos de ensaios de poluição ambiental e o uso obrigatório de EPIs.

Palavras-Chave: Mineração, Amianto, Asbestose, Riscos Tecnológicos Ambientais, Saúde do Trabalhador.

\section{INTRODUÇÃO}

O uso em carga escala do amianto na indústria brasileira tem gerado muitas controvérsias e a tentativa de mascarar os problemas de ordem ambiental e ocupacional referente à mineração do mineral que apresenta "diferentes fibras de amianto, tanto do grupo dos anfibólios (amosita, crocidolita, antofilita, actinolita e tremolita) quanto das serpentinas (crisotila)" (WUNSCH FILHO; NEVES; MONCAU, 2001, p. 1).

Atualmente existe o esforço de adequação e expansão dos Sistemas de Vigilância e Informação em Saúde Ocupacional e Ambiental dos estados brasileiros para a proteção dos trabalhadores que trabalham com a mineração de amianto. A fiscalização é complexa na medida em que tal ação muitas vezes é realizada clandestinamente. Embora, os Estados de São Paulo e Rio de Janeiro tenham determinado o fim da exploração do amianto, muitos municípios brasileiros continuam mantendo a exploração do mineral e os resultados na saúde do trabalhador são visivelmente as doenças e a incapacidade física, as mortes por

RC: 87951

Disponível em: https://www.nucleodoconhecimento.com.br/engenhariaambiental/tecnologicos-ambientais 
neoplasias pulmonares por silicose e outras doenças (BARCELLOS; QUITÉRIO, 2006).

A problemática de pesquisa aponta a seguinte análise: Como tornar a atuação da vigilância ambiental no campo das políticas públicas de saúde nas demandas referentes ao trabalho de mineradores de amianto?

Os pressupostos do estudo apontam que as alternativas mais eficientes poderão ser o aumento regular da fiscalização em regiões de mineração, maior acesso ao atendimento à saúde ocupacional, expansão de procedimentos em exames para a conclusão de diagnósticos e a regulação da atividade de forma a determinar medidas que eliminem os ricos por meio de uso de Equipamentos de Proteção Individual - EPIs (CONFEDERAÇÃO NACIONAL DOS TRABALHADORES NAS INDÚSTRIAS - CNTI, 2013).

A finalidade desse artigo é demonstrar os riscos de natureza tecnológica e ambiental que se apresentam nas atividades produtivas contemporâneas da mineração de amianto. Pretende-se demonstrar os riscos iminentes das operações, os efeitos no organismo humano em trabalhadores que estiveram exposições prolongadas; demonstrar as doenças ocupacionais que decorrem da exposição contínua ao mineral.

Justifica-se a escolha do tema em face da preocupação com a Segurança e Saúde do Trabalho, considerando-se que o diagnóstico das doenças causadas pelo mineral é de complexo, além de haver raras notificações de doenças ocupacionais nesse campo de produção.

A relevância do estudo é demonstrar a importância da Segurança e Saúde do Trabalho para a eficiência de processos de padronização de estruturas de intervenção no ambiente de trabalho de mineradores em face dos interesses de

RC: 87951

Disponível em: https://www.nucleodoconhecimento.com.br/engenhariaambiental/tecnologicos-ambientais 
natureza econômica no mercado devido ao valor do amianto e seu vasto uso na indústria.

$\mathrm{Na}$ atividade laboral existe uma grande diversidade de riscos ocupacionais que exigem por parte da saúde pública e das empresas responsabilidade distintas no campo da Segurança e Saúde do Trabalho e higiene do Trabalho como ciências que atuam diretamente na pesquisa e na orientação de situações de risco diante dos desafios colocados pelo sistema de produção.

\section{DESENVOLVIMENTO}

\subsection{MINERAÇÃO DO AMIANTO: UMA ABORDAGEM AOS RISCOS TECNOLÓGICOS AMBIENTAIS E SAÚDE DO TRABALHO}

Partindo-se da mineração do amianto e dos riscos tecnológicos ambientais a sociedade atualmente vivencia-se uma constante vulnerabilidade expressa pelo agravamento dos acidentes e doenças de natureza ocupacional que nem mesmo as diretrizes de segurança algumas vezes não são suficientes diante dos problemas tecnológicos ambientais gerados pela produção.

Dentro dessa abordagem da vulnerabilidade, as diretrizes de Segurança e Saúde do Trabalhador tem buscado apresentar propostas de caráter integrador com o objetivo de suplantar procedimentos fragmentários, a partir de fundamentos teóricometodológicos nas técnicas de análise de risco em uma abordagem interdisciplinaridade e sistêmica, que somente apresentam resultados eficazes mediante a plena participação do trabalhador nas atividades de controle dos riscos tecnológicos ambientais (CONFEDERAÇÃO NACIONAL DOS TRABALHADORES NAS INDÚSTRIAS - CNTI, 2013).

Nesse contexto da segurança e saúde do trabalho, se firmado a definição de riscos tecnológicos ambientais, relacionados aos efeitos químico-radioativos que produzem

RC: 87951

Disponível em: https://www.nucleodoconhecimento.com.br/engenhariaambiental/tecnologicos-ambientais 
grandes riscos nas atividades produtivas, tendo como foco a análise abrangente "dos perigos da tecnologia e suas dimensões técnicas, cognitiva, social, cultural e filosófica" (PORTO; FREITAS, 1997, p. 60).

A responsabilidade dos órgãos de proteção à saúde do trabalhador de dar o devido suporte à Política Nacional de Segurança e Saúde do Trabalhador que seja efetivamente cumprida, sob o ponto de vista dos riscos tecnológicos ambientais provocadores de doenças ocupacionais em todos os sistemas produtivos com o objetivo de determinar uma interface entre segurança/higiene/saúde e a dimensão ambiental no trabalho por meio de procedimentos padronizados (MARANO, 2007).

$\mathrm{Na}$ mineração de amianto os estudos de abordagem epidemiológica demonstram o grande risco em potencial se constitui na doença pleural obtida por exposição, cuja prevalência elevada chega a $30 \%$ das populações mineradoras em cortes de estudo de até 10 anos que demonstraram a prevalência de doenças como "o câncer de laringe e alguns tumores gastrointestinais também foram relacionados ao amianto em alguns estudos" (WUNSCH FILHO; NEVES; MONCAU, 2001, p. 1).

Sob esse ponto de vista, as atividades laborais são estudadas e analisadas sob cinco áreas com enfoques tecnológico ambiental.

O amianto é um mineral encontrado facilmente na natureza e que por suas características peculiares como, flexibilidade e resistência tênsil elevadas às propriedades química, térmica e elétrica são utilizados comercialmente em vários produtos, tais como o cimento cujas propriedades fazem do amianto um mineral usado como matéria-prima para a constituição de outros elementos, desde aqueles ligados aos componentes da construção civil, como cimento, telhas, caixa d'agua, como também nas indústrias automotivas como lonas, freios e revestimentos de discos de embreagem.

$\mathrm{RC}: 87951$

Disponível em: https://www.nucleodoconhecimento.com.br/engenhariaambiental/tecnologicos-ambientais 
Conforme Martines e Grando (2008, p. 01) devido às propriedades do amianto e entre elas principalmente a sua resistência, este mineral é utilizado no Brasil e no mundo na fabricação de diferentes produtos comerciais. $O$ autor refere ainda que estaticamente aproximadamente $85 \%$ são utilizados pelas indústrias de cimento amianto ou fibrocimento, $10 \%$ na industrialização de autopeças, 3\% em produtos têxteis e $2 \%$ em fabricação de produtos químicos/plásticos.

Entretanto, apesar destes benefícios químicos e físicos, o amianto é formado por elementos que comprovadamente podem produzir problemas de saúde, como doenças pulmonares e câncer.

Para Medina (2008, p. 02) algumas pesquisas já realizadas comprovaram que o amianto é um mineral, cujos elementos químicos que o compõem apresentam potencial cancerígeno. Estes elementos de potencial cancerígeno podem ser detectados em qualquer momento de produção, transformação ou mesmo uso do mineral.

A autora refere ainda que conforme a Organização Mundial de Saúde (OMS), a crisotila, elemento associado ao amianto, pode ser um fator desencadeante de inúmeras patologias pulmonares, entre elas a asbestose, o câncer pulmonar e mesotelioma de pleura e peritônio. Além disso, pesquisas revelam que não existe nenhum limite seguro de exposição para o risco carcinogênico proporcionado por este componente químico.

Em relação à Asbestose, de acordo com Martines e Grando (2008, p. 02) considerase que:

é uma doença pulmonar relacionada à prolongada inalação de poeira contendo alta concentração de fibras de amianto. As fibras alojam-se nos alvéolos pulmonares, e, para se defender, o organismo deposita sobre elas uma proteína semelhante a um "cimento" que cicatriza o alvéolo, impedindo que se encha de ar. Este processo, repetindo-se ao longo dos anos, pode tornar o pulmão fibrosado e sem elasticidade, com dificuldades respiratórias.

RC: 87951

Disponível em: https://www.nucleodoconhecimento.com.br/engenharia- 
No que concerne as outras patologias causadas pelo contato com o amianto temos que nos casos de aparecimento de câncer de pulmão, os mesmos são mais comuns quando há uma associação entre fumo e a substância cancerígena. Além disso, conforme Martines e Grando (2008, p. 02) considera-se que entre o inicio de exposição aos elementos do amianto até o surgimento do quadro clínico de câncer de pulmão tem-se um período de tempo de aproximadamente 20 anos.

\subsection{O GERENCIAMENTO DE RISCO EM AMBIENTES DE MINERAÇÃO}

O gerenciamento de risco é uma ação sistemática nas organizações que desenvolvem novos modelos de análise de risco buscando identificar, na complexidade dos eventos tecnológicos ambientais do trabalho, os fatores que os desencadeiam simultaneamente, as condições de risco potencial preexistentes no trabalho (SOUZA; FONTES, 2008).

Veloso Neto (2013) é adepto da aplicação de sistema de Gestão de Segurança nas mineradoras de amianto. O gerenciamento de risco incorpora necessidade de inspeção periódica dos locais de trabalho objetivando a avaliação do uso de EPIs pelos trabalhadores, bem como o tempo de exposição baseada na redução de tempo de serviço.

Nesse contexto, cabe à empresa mineradora a contratação de um especialista para gerenciar a remoção adequada do amianto para que sejam observadas as regras de segurança de acordo com a NR - 15 (anexo 12) que regulamenta o uso na forma de fabricação do produto, sendo proibido o uso de alguns tipos de amianto.

A normalização técnica, NBR 5643 (MB1090) regulamenta o fabrico de telhas em amianto, a NBR13956-1 que regulamenta o processo de exposição do trabalhador aos ambientes onde existem riscos de sílica determinando as diretrizes de análise por meio de ensaios do nível de sílica no ambiente de mineração.

RC: 87951

Disponível em: https://www.nucleodoconhecimento.com.br/engenhariaambiental/tecnologicos-ambientais 
As outras normas técnicas que se encontram no quadro abaixo se referem às análises ambientais referentes aos métodos de ensaios para poluição ambiental e concentração de substâncias químicas:

Quadro 1: Normas Técnicas ambientais que podem se enquadrar no uso de amianto pelas mineradoras.

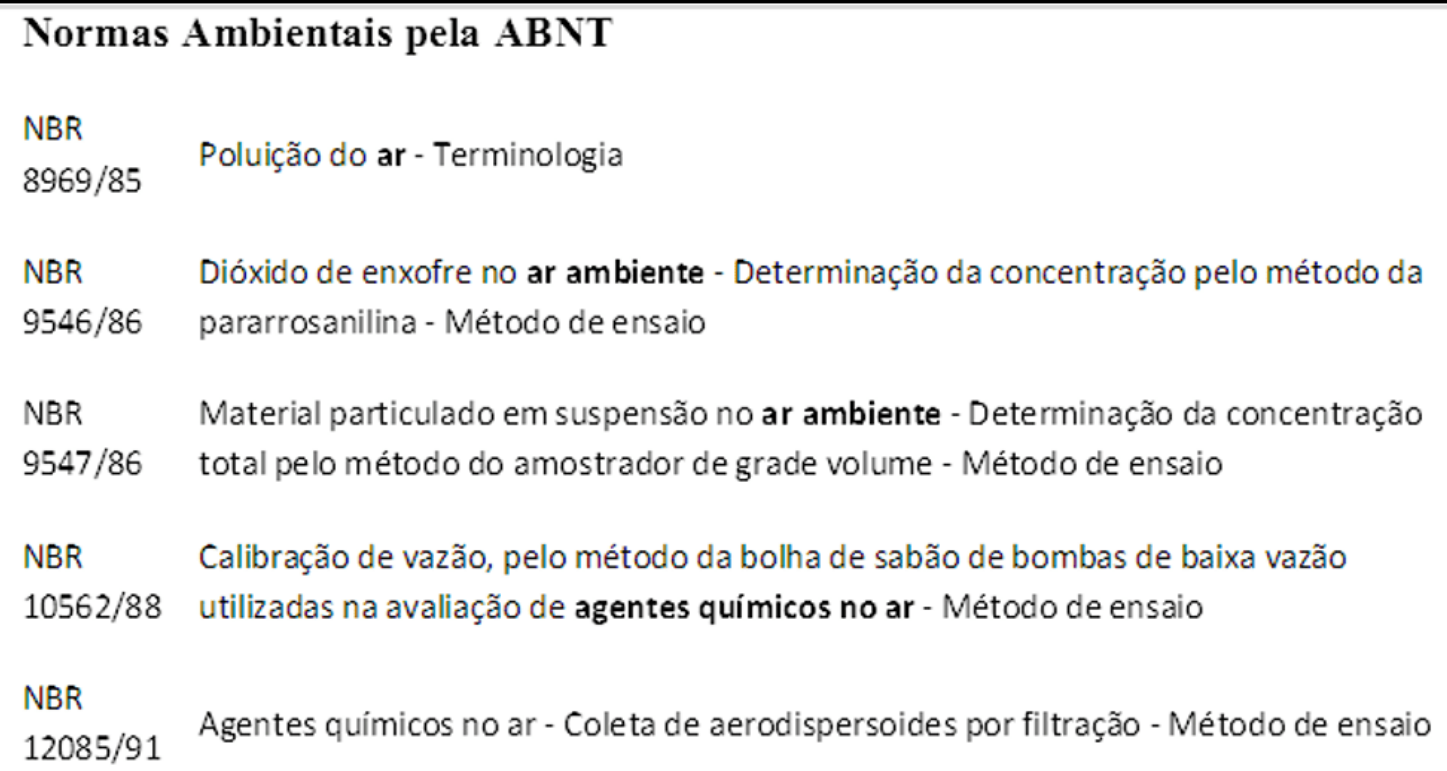

Fonte: Veloso Neto (2013)

Tavares (2004) analisa que os processos produtivos potencialmente perigosos a exemplo de ações realizadas por trabalhadores em ambientes de mineração de amianto, em atmosferas potencialmente perigosas devido a sílica favorecem as probabilidades reais de riscos diante das forças de produção que colocam em risco o ser humano.

RC: 87951

Disponível em: https://www.nucleodoconhecimento.com.br/engenhariaambiental/tecnologicos-ambientais 
Quadro 2: Gerenciamento de riscos

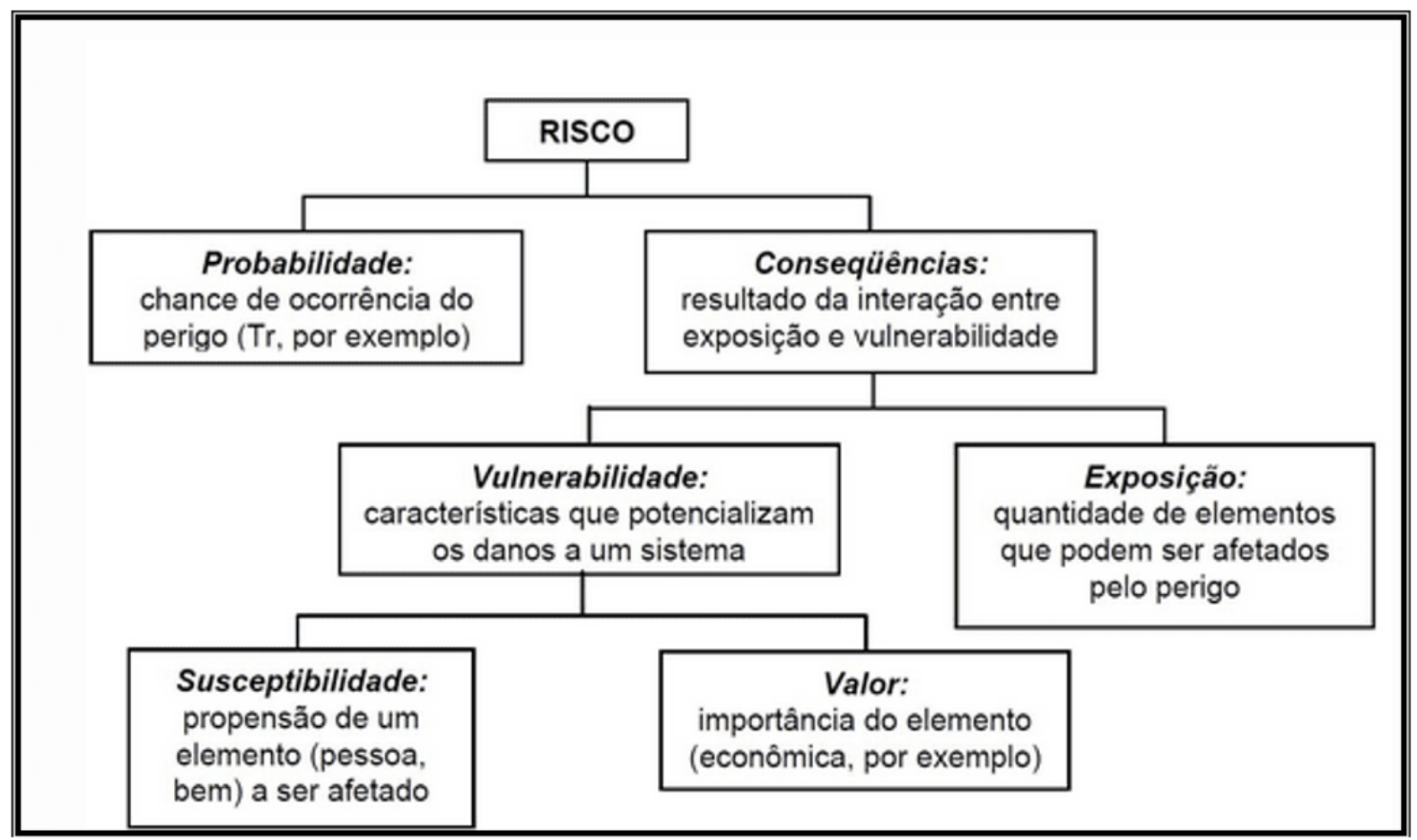

Fonte: Marano (2007)

Como se evidencia, a aplicação das orientações de normas regulamentoras favorecem as instruções no trabalho em termos de segurança e saúde, bem como as medidas de prevenção nos diversos ambientes de trabalho e atividades produtivas que envolvem o manuseio do amianto.

Os eventos perigosos à exposição ao amianto poderá causar no trabalhador uma reação adversa. A severidade do evento é vista como consequência do ano gerado que podem lesões, traumas, morte, redução de capacidade para o trabalho, etc.

O gerenciamento de risco em mineração de amianto implica na definição de risco que envolve dois importantes elementos: A probabilidade de um evento de risco e a consequência perigosa desse evento caracterizado pela prevalência de doenças ocupacionais em médio e longo prazo.

RC: 87951

Disponível em: https://www.nucleodoconhecimento.com.br/engenharia- 
No que concerne a esse contexto legal e operacional do uso do amianto, tem-se de acordo com Martines e Brando (2008, p. 03) refere que existe o denominado Critério de Saúde ambiental 15 lançado pela Organização Mundial de Saúde. Por meio deste critério, tem-se a orientação que em relação às fibras biopersistentes do amianto devem passar por um processo de controle rígido e permanente com o intuito de minimizar os efeitos tóxicos e carcinogênicos.

Em se tratando de eventos de riscos, estes são provenientes de atividades produzidas colocando em risco a saúde e a integridade física do trabalhador. Esses eventos decorrem do alto grau de riscos da extração mineral do amianto, associadas às situações de desinformação e carência de fiscalização.

Apesar de todos os malefícios causados pelo uso do amianto pelas indústrias ainda não existem Leis específicas de proibição da extração, com exceção de alguns estados brasileiros como São Paulo e Rio de Janeiro, em outras situações de uso, a maioria dos Estados estabeleçam critérios de controle de exploração do amianto, determinando o uso de EPIs.

Sabe-se que várias patologias são causadas pelos elementos formadores do amianto e que estas patologias não acometem somente os trabalhadores de mineração, mas também os que atuam nas indústrias e fabricas, mas também toda a população que reside próximo do ambiente produtor.

Considera-se assim que a luta pelo uso do seguro do amianto pelas indústrias deve ser de todos, trabalhadores e sociedade, que possuem o direito de viver em um ambiente saudável e risco de danos.

\section{CONSIDERAÇÕES FINAIS}

O estudo permitiu evidenciar as dificuldades de proibição do uso do amianto na indústria brasileiro. O seu uso em larga escala em amplos setores de produção

RC: 87951

Disponível em: https://www.nucleodoconhecimento.com.br/engenhariaambiental/tecnologicos-ambientais 
determina o seu uso aplicando-se medidas restritivas e limites de exposição do trabalhador. Mesmo assim, essa questão é ainda polêmica, considerando que São Paulo e Rio de Janeiro aboliram o uso do amianto diante dos efeitos negativos na saúde dos trabalhadores.

A Confederação Nacional dos Trabalhadores da Indústria - CNTI tem buscado alternativas diante da importância do amianto na economia nacional cujas tendências são a extração controlada e com inspeção por meio de métodos de ensaios de poluição ambiental e o uso obrigatório de EPIs.

Atualmente a prevenção quanto aos riscos tecnológicos ambientais induzem à sociedade a busca de alternativas para evitar a vulnerabilidade dos trabalhadores durante o processo produtivo, a partir de gerenciamento de risco que possam reduzir ao máximo o potencial nefasto dos riscos da extração e amianto.

A manutenção das atividades de extração sem as devida precauções das normas técnicas colocam o trabalhador em grande vulnerabilidade com doenças ocupacionais que no que concerne ao número de casos patológicos que tem vitimado os trabalhadores, embora atualmente não existam dados precisos, as pesquisas referentes aos de 1980 e 2001 demonstram os resultados epidemiológicos acerca das doenças ocupacionais e seus graves riscos.

A Constituição Brasileira determinou o pleno direito do trabalhador de viver em um ambiente laboral saudável e livre de riscos a saúde, sem a projeção de qualquer tipo de problemas tecnológicos ambientais gerados pela produção.

Dentro dessa abordagem da vulnerabilidade demonstrou-se a necessidade do gerenciamento de risco, inspeção periódica e criação de uma equipe de profissionais para informar devidamente os riscos aos trabalhadores.

RC: 87951

Disponível em: https://www.nucleodoconhecimento.com.br/engenhariaambiental/tecnologicos-ambientais 


\section{REFERÊNCIAS}

BARCELOS, Christovam; QUITÉRIO, Luiz Antônio Dias. Vigilância ambiental em saúde e sua implantação no Sistema único de Saúde. Revista de Saúde Pública, v. São Paulo, n. 01, ed. 40, jun./fev. 2006.

CONFEDERAÇÃO Nacional dos Trabalhadores nas Indústrias - CNTI. Acordo nacional para extração, beneficiamento e utilização segura e responsável do amianto crisotila 2013 a 2015. Disponível em: <http://www.sinticomex.org.br/ckfinder/userfiles/files/ACT\%20CNTA\%202015.pdf>. Acesso em: 5 mar. 2021.

MARANO, Vicente Pedro. Doenças ocupacionais. 2. ed. São Paulo: LTr, 2007.

MARTINES, Marco Utrera; BRANDO, Daniela. Amianto: proibição, uso controlado ou imobilização? 2008.

Disponível

em:

<http://ambientes.ambientebrasil.com.br/residuos/artigos/amianto\%3A_proibicao,_us o_controlado_ou_imobilizacao\%3F.html> Acesso em: 6 mar. 2021.

MEDINA, Damares. O Amianto e as Restrições ao seu uso na Jurisprudência do Supremo Tribunal Federal. Revista Prática Jurídica, São Paulo, v. VII, n. 72, mar. de 2008.

SOUZA, Luis Cláudio Paiva de; FONTES, Carlos Eduardo Mazzuco. Qualidade de vida no trabalho: saúde emocional e gestão estratégica. São Paulo: EDICON, 2008

TAVARES, José da Cunha. Noções de prevenção e controle de perdas em segurança do trabalho. São Paulo: SENAC, 2004.

VELOSO NETO, Hernani. Construção social do risco e da segurança do trabalho em contexto organizacional. Rio de Janeiro: Civeri Publishing, 2013.

RC: 87951

Disponível em: https://www.nucleodoconhecimento.com.br/engenhariaambiental/tecnologicos-ambientais 
WUNSCH FILHO; V. NEVES, H.; MONCAU, J. E. Amianto no Brasil: conflitos científicos e econômicos. Revista Associação Médica Brasileira, São Paulo, v. 47, n. 3, jul./set. 2001.

Enviado: Abril, 2021.

Aprovado: Junho, 2021.

RC: 87951

Disponível em: https://www.nucleodoconhecimento.com.br/engenhariaambiental/tecnologicos-ambientais 